\title{
Intracellular iron sensing by the direct binding of iron to regulators
}

\author{
Takanori Kobayashi ${ }^{1,2}$ and Naoko K. Nishizawa ${ }^{2 *}$ \\ ${ }^{1}$ Japan Science and Technology Agency, PRESTO, Kawaguchi, Japan, ${ }^{2}$ Research Institute for Bioresources and \\ Biotechnology, Ishikawa Prefectural University, Nonoichi, Japan
}

Keywords: iron deficiency, IDEF1, HRZs/BTS, sensor, signaling

\section{Introduction}

Iron $(\mathrm{Fe})$ is an essential micronutrient for virtually all living organisms; it is incorporated into numerous proteins as Fe-sulfur (S) clusters, heme, or free Fe, and it mediates many metabolic processes, including photosynthesis and cellular respiration. Due to the low solubility and high reactivity of $\mathrm{Fe}$, living cells must tightly regulate Fe acquisition in response to changes in Fe availability in the environment. The molecular components involved in Fe acquisition and its regulation have been studied extensively in plants, animals, fungi, and bacteria, revealing specific mechanisms in each kingdom. Fe acquisition in higher plants is mediated by a chelation-based pathway using mugineic acid family phytosiderophores (Strategy II) or a reduction-based pathway using ferric-chelate reductases (Strategy I), depending on the species (Römheld and Marschner, 1986).

OPEN ACCESS

Edited by:

Olena Vatamaniuk,

Cornell University, USA

Reviewed by:

Sebastien Thomine,

Centre National de la Recherche

Scientifique, France

Wolfgang Schmidt,

Academia Sinica, Taiwan

*Correspondence:

Naoko K. Nishizawa, annaoko@mail.ecc.u-tokyo.ac.jp

Specialty section: This article was submitted to Plant Traffic and Transport, a section of the journal Frontiers in Plant Science

Received: 10 January 2015 Paper pending published: 05 February 2015 Accepted: 26 February 2015 Published: 11 March 2015

Citation: Kobayashi T and Nishizawa NK (2015) Intracellular iron sensing by the direct binding of iron to regulators.

Front. Plant Sci. 6:155. doi: $10.3389 /$ fpls.2015.00155
Molecular components involved in both pathways are induced under Fe deficiency and repressed under Fe sufficiency, primarily at the transcript level; however, protein-level regulation has also been reported for some components (Lan et al., 2011; Kobayashi and Nishizawa, 2012). To achieve expressional regulation based on Fe availability, Fe sensing systems must exist, but the signals and sensors have not been clarified in plants. Although various metabolites affected by Fe availability are thought to influence Fe deficiency responses, we propose that the direct binding of Fe to expressional regulators is the primary Fe sensing event in plant cells, similar to the mechanisms in animals, fungi, and bacteria. The recent identification of the Fe-binding regulators IDEF1 and HRZs/BTS in plants supports this hypothesis.

\section{Intracellular Fe Sensing in Animals, Fungi, and Bacteria}

In animals, Fe homeostasis is mediated by complicated transcriptional and post-transcriptional regulatory networks. Among them, the Fe regulatory protein (IRP)/Fe-responsive element (IRE) system plays a principal role in the cellular response to Fe (Anderson et al., 2012). Under Fe deficiency IRP1 and IRP2 bind to IREs, stem-loop structures present in various mRNAs that are involved in Fe homeostasis. IRP binding to an IRE increases the stability of mRNAs for Fe acquisition such as transferrin receptor, and inhibits the translation of proteins for Fe sequestration such as ferritin. Under Fe-sufficient conditions, IRP-IRE binding becomes less dominant because of two Fe-sensing mechanisms. First, an Fe-S cluster binds to IRP1 and converts it to cytosolic aconitase, inhibiting its mRNA binding activity. Second, IRP2 is ubiquitinated and subjected to proteasomal degradation via the function of F-box leucine-rich repeat protein 5 (FBXL5). Fe binding to FBXL5 triggers IRP2 degradation, while the Fe-free form of FBXL5 is rapidly degraded. These two mechanisms ensure preferential IRP-IRE binding under Fe deficiency. Animal cells possess additional Fe-sensing mechanisms, including abolishment of the hypoxia-inducible factor (HIF) regulatory pathway by Fe-requiring HIF prolyl hydroxylases (Thompson and Bruick, 2012) and de-repression of Bachmediated transcriptional regulation by heme-Bach binding (Igarashi and Watanabe-Matsui, 2014). 
In Saccharomyces cerevisiae, the transcription factors Aft 1 and Aft 2 are major players in the Fe deficiency response. Aft $1 / 2$ nuclear function is inhibited by an Fe-replete signal generated in mitochondria via Fe-S clusters, the cytosolic monothiol glutaredoxins Grx3 and Grx4, and the BolA-like protein Fra2. These proteins and $\mathrm{Fe}-\mathrm{S}$ clusters form a cytosolic complex that enters the nucleus and dissociates Aft1 and Aft2 from their target cisacting elements (Ueta et al., 2012; Poor et al., 2014). Thus, Fe-S clusters are primary signaling molecules for Fe deficiency responses in yeast cells.

Bacteria sense Fe by binding directly to members of the ferric uptake regulator (Fur) family (Lee and Helmann, 2007). Fur from Escherichia coli is a transcriptional repressor that binds to cis-acting elements and ferrous Fe. When cellular Fe is scarce, Fur does not bind to Fe; this decreases the affinity between Fur and its cis-acting elements, releasing the repression of Fe uptake-related genes.

Despite the diverse molecular mechanisms for Fe acquisition and regulation across kingdoms, all of the above-mentioned regulatory events are mediated by the direct binding of $\mathrm{Fe}$ or $\mathrm{Fe}$-containing prosthetic groups (Fe-S clusters and heme) to regulatory proteins. Such direct $\mathrm{Fe}$ sensing is thought to be advantageous in rapidity and accuracy compared with indirect Fe sensing through metabolic changes, though the latter would also be important for fine-tuning the response.

\section{Fe-Binding Regulators in Plants}

Two types of Fe-binding regulators for Fe deficiency responses are known in higher plants: IDEF1 and HRZs/BTS (Figure 1A). IDEF1 is a Gramineae-specific transcription factor that positively regulates various genes involved in Fe uptake and translocation (Kobayashi et al., 2007, 2009). Analyses using transgenic rice plants revealed that IDEF1 is especially important for the early response to Fe deficiency. Nevertheless, IDEF1 transcription is not induced in response to Fe deficiency, suggesting that IDEF1 regulates Fe deficiency responses by receiving a signal. IDEF1 possesses characteristic histidine-asparagine repeats and proline-rich regions that bind ferrous $\mathrm{Fe}$ and other divalent metals reversibly (Figure 1A; Kobayashi et al., 2012). These metal-binding regions are essential for overexpressed IDEF1 to enhance Fe deficiency responses at the early stages of Fe deficiency, although a direct relationship between metal binding and IDEF1 function has not been proven.

The second group of Fe-binding regulators in plants is HRZs/BTS; its members have been designated OsHRZ1 and OsHRZ2 in rice (Kobayashi et al., 2013) and BTS in Arabidopsis thaliana (Long et al., 2010). OsHRZ1, OsHRZ2, and BTS are transcriptionally induced under Fe deficiency, and their homologs are widely conserved among plants and algae (Urzica et al., 2012; Kobayashi et al., 2013). HRZs/BTS contain hemerythrin domains, three zinc ( $\mathrm{Zn}$ ) finger domains, including a RING finger, and a rubredoxin-type fold in each protein (Figure 1A). The hemerythrin domain binds ferrous $\mathrm{Fe}$ and molecular oxygen in animals and bacteria (Stenkamp, 1994), and is present in human FBXL5 as an Fe-binding and -sensing domain (Salahudeen et al., 2009; Vashisht et al., 2009; Thompson et al., 2012). In vitro studies
A
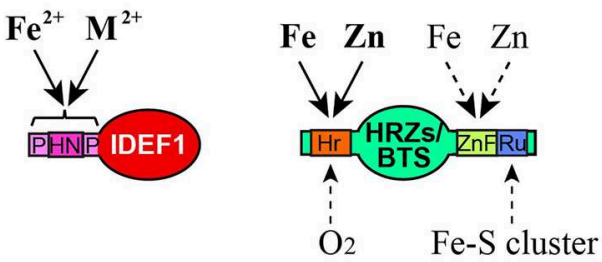

B

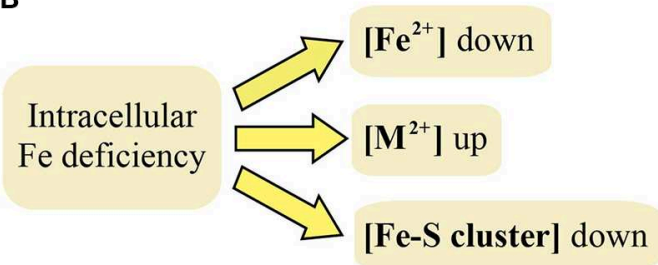

FIGURE 1 | Possible Fe signals recognized by IDEF1 and HRZs/BTS.

(A) Known or putative binding of $\mathrm{Fe}$ and related molecules to domains of IDEF1 and HRZs/BTS. Solid and broken lines indicate known and putative bindings, respectively. $\mathrm{M}^{2+}$, divalent metal ions such as $\mathrm{Zn}$, copper and nickel. $\mathrm{HN}$, histidine-asparagine repeat; $\mathrm{P}$, proline-rich region; $\mathrm{Hr}$, hemerythrin domains; ZnF, Zn finger domains; Ru, rubredoxin-type fold. (B) Deduced effects of intracellular Fe deficiency on concentrations of $\mathrm{Fe}$ and related molecules which may act as Fe signals.

revealed that the N-terminal regions of OsHRZ1, OsHRZ2, and BTS containing hemerythrin domains bind Fe as well as $\mathrm{Zn}$ (Kobayashi et al., 2013; Selote et al., 2015). OsHRZ1, OsHRZ2, and BTS are negative regulators of Fe deficiency responses; their knockdown results in enhanced tolerance to Fe deficiency and $\mathrm{Fe}$ accumulation in rice and Arabidopsis (Long et al., 2010; Kobayashi et al., 2013; Selote et al., 2015). OsHRZ1, OsHRZ2, and BTS also possess ubiquitination activity, which is likely attributed to the RING finger domain (Kobayashi et al., 2013). Selote et al. (2015) demonstrated that BTS binds and destabilizes two Arabidopsis basic helix-loop-helix (bHLH) proteins, ILR3 and AtbHLH115, most likely through proteasome-mediated degradation following ubiquitination. ILR3 and AtbHLH115 bind to another Arabidopsis bHLH protein, PYE, which regulates various genes involved in Fe homeostasis (Long et al., 2010). ILR3 regulates the expression of the Arabidopsis vacuolar Fe transporterlike genes AtVTL1, AtVTL2, and AtVTL5, which are thought to sequester $\mathrm{Fe}$ into vacuoles under Fe-replete conditions (Rampey et al., 2006; Gollhofer et al., 2014). These results suggest that BTS regulates Fe homeostasis via the ubiquitination of ILR3 and AtbHLH115, and modulation of the PYE-mediated Fe deficiency response; however, the precise functions of ILR3 and AtbHLH115 in Fe homeostasis are unclear. The ubiquitination targets of rice HRZs have not been identified, although bHLH proteins similar to PYE, ILR3, and AtbHLH115 are present in rice.

A common property of IDEF1 and HRZs/BTS is their ability to bind non-Fe metals, including $\mathrm{Zn}$, which compete with $\mathrm{Fe}$ for binding (Figure 1A; Kobayashi et al., 2012, 2013). This suggests that Fe sensing in plant cells is mediated either by Fe itself, 
non-Fe metals, or both. Non-Fe metals might act agonistically or antagonistically. The former case has been demonstrated for bacterial Fur, which is able to bind manganese in place of Fe, acting similarly to the Fe-bound form (Lee and Helmann, 2007). However, the antagonistic function of non-Fe metals might be more plausible in plant cells because the concentrations of non-Fe metals in plant tissues increase during Fe deficiency (Figure 1B). Recently, dynamic imaging by FRET sensors enabled an estimation of cytosolic concentration of free $\mathrm{Zn}^{2+}$ in Arabidopsis roots (Lanquar et al., 2014). However, the concentrations of free Fe remain unknown in plant cells. Monitoring the free concentrations of $\mathrm{Fe}$ and other metals in plant cells under various nutrient conditions would be important for clarifying $\mathrm{Fe}$ sensing by these metals. Interestingly, the subcellular localization of the Arabidopsis transporter IRT1, which is involved in the uptake of ferrous $\mathrm{Fe}$ and other divalent metals by roots, is regulated by non-Fe metals, but not by $\mathrm{Fe}$ (Barberon et al., 2014). IRT1 shuttles between the plasma membrane and early endosome/trans-Golgi network dependent on monoubiquitination; however, the depletion of non-Fe metals stabilizes IRT1 localization at the root surface. This regulation is thought to be a protective mechanism to prevent excess uptake of non-Fe metals such as $\mathrm{Zn}$ and manganese (Thomine and Vert, 2013). This regulation also contributes to Fe homeostasis, thus it suggests the physiological suitability of non-Fe metals as a type of $\mathrm{Fe}$ signal.

Another suggested property of HRZs/BTS is sensing multiple Fe-related signals (Figure 1). In addition to binding $\mathrm{Fe}$ and $\mathrm{Zn}$, the hemerythrin domains of HRZs/BTS may bind molecular oxygen, because these domains bind oxygen coordinated with $\mathrm{Fe}$ in invertebrates (Stenkamp, 1994). Human FBXL5 is destabilized under hypoxia as well as under Fe deficiency, suggesting tight linkage between Fe deficiency responses and cellular redox status (Salahudeen et al., 2009; Vashisht et al., 2009; Thompson et al., 2012). HRZs/BTS possess additional domains that are potentially involved in Fe sensing, including $\mathrm{Zn}$ fingers and rubredoxin-type folds. The coordination of $\mathrm{Zn}$ to $\mathrm{Zn}$ fingers usually contributes to protein structure, but some $\mathrm{Zn}$ fingers are known to function as $\mathrm{Zn}$ sensors (Bird et al., 2003). Fe also binds to some Zn fingers (Conte et al., 1996; Besold et al., 2010). Rubredoxintype folds in bacteria bind Fe-S clusters, which are important Fe signaling molecules in animals and yeast. These observations suggest that HRZs/BTS sense and coordinate more than one type of Fe signal. Although the mechanisms underlying the regulation of Fe homeostasis are thought to be distinct between plants and other organisms, the identification of HRZs/BTS suggests the partial conservation of Fe-sensing mechanisms across kingdoms.

\section{References}

Anderson, C. P., Shen, M., Eisenstein, R. S., and Leibold, E. A. (2012). Mammalian iron metabolism and its control by iron regulatory proteins. Biochim. Biophys. Acta 1823, 1468-1483. doi: 10.1016/j.bbamcr.2012.05.010

Barberon, M., Dubeaux, G., Kolb, C., Isono, E., Zelazny, E., and Vert, G. (2014). Polarization of IRON-REGULATED TRANSPORTER 1 (IRT1) to the

\section{Identifying Direct Fe Sensors in Plants}

Recently, we proposed that a cellular Fe sensor must meet the following three criteria: (i) bind to $\mathrm{Fe}$ or intimately related molecule(s), (ii) change its function in response to binding, and (iii) regulate Fe homeostasis (Kobayashi and Nishizawa, 2014). Both IDEF1 and HRZs/BTS bind Fe and Zn, and they regulate Fe deficiency responses, satisfying criteria (i) and (iii), but there is no clear evidence to support criterion (ii). Very recently, Selote et al. (2015) reported that BTS stability is dependent on Fe, providing evidence linked to criterion (ii). They showed that BTS produced in vitro using a wheat germ extract system was less abundant when $\mathrm{Fe}$ was included in the translation reaction mixture. This effect was abolished by a point mutation at a putative Fe-binding residue in the hemerythrin domain, suggesting that Fe binding to the hemerythrin domain destabilizes and inhibits the function of BTS. When expressed in planta, deletion of the hemerythrin domains from BTS resulted in increased protein stability. However, complementation tests using an Arabidopsis bts knockdown mutant revealed that a truncated version of BTS without the hemerythrin domains functioned more or less similarly to full-length BTS with respect to physiological Fe deficiency responses (Selote et al., 2015), suggesting the limited importance of Fe-mediated BTS degradation via the hemerythrin domains. Further characterization of the mechanisms underlying the degradation and function of HRZs/BTS in relation to the binding of $\mathrm{Fe}, \mathrm{Zn}$, oxygen, or Fe-S clusters is essential to identify whether these regulators are central Fe sensors that determine downstream responses to Fe deficiency. Similarly, identification of the molecular mechanism by which IDEF1 alters its function due to $\mathrm{Fe}$ or $\mathrm{Zn}$ binding is needed. It is possible that primary Fe sensing in plant cells is established by unknown factors, while IDEF1 and HRZs/BTS modulate these events and mediate appropriate Fe deficiency responses. In this scenario, the identification of a central regulator that changes its function in response to binding $\mathrm{Fe}$ or an intimately related molecule would be key to verifying it as a central Fe sensor.

\section{Acknowledgments}

We thank Dr. Takeshi Senoura (Ishikawa Prefectural University, Japan) for valuable discussions and critically reading the manuscript. Our studies of Fe sensing are supported by the Japan Science and Technology Agency program PRESTO (to TK) and, in part, by the Japan Science and Technology Agency program ALCA (to NN) and a Grant-in-Aid from the Ministry of Education, Culture, Sports, Science, and Technology (23248011, to $\mathrm{NN}$ ).

plant-soil interface plays crucial role in metal homeostasis. Proc. Natl. Acad. Sci. U.S.A. 111, 8293-8298. doi: 10.1073/pnas.1402262111

Besold, A. N., Lee, S. J., Michel, S. L., Sue, N. L., and Cymet, H. J. (2010). Functional characterization of iron-substituted neural zinc finger factor 1: metal and DNA binding. J. Biol. Inorg. Chem. 15, 583-590. doi: 10.1007/s00775-010-0626-1

Bird, A. J., McCall, K., Kramer, M., Blankman, E., Winge, D. R., and Eide, D. J. (2003). Zinc fingers can act as $\mathrm{Zn}^{2+}$ sensors to regulate transcriptional 
activation domain function. EMBO J. 22, 5137-5146. doi: 10.1093/emboj/ cdg484

Conte, D., Narindrasorasak, S., and Sarkar, B. (1996). In vivo and in vitro ironreplaced zinc finger generates free radicals and causes DNA damage. J. Biol. Chem. 271, 5125-5130. doi: 10.1074/jbc.271.9.5125

Gollhofer, J., Timofeev, R., Lan, P., Schmidt, W., and Buckhout, T. J. (2014). Vacuolar-iron-transporter1-like proteins mediate iron homeostasis in Arabidopsis. PLoS ONE 9:e110468. doi: 10.1371/journal.pone.0110468

Igarashi, K., and Watanabe-Matsui, M. (2014). Wearing red for signaling: the heme-Bach axis in heme metabolism, oxidative stress response and iron immunology. Tohoku J. Exp. Med. 232, 229-253. doi: 10.1620/tjem.232.229

Kobayashi, T., Itai, R. N., Aung, M. S., Senoura, T., Nakanishi, H., and Nishizawa, N. K. (2012). The rice transcription factor IDEF1 directly binds to iron and other divalent metals for sensing cellular iron status. Plant J. 69, 81-91. doi: 10.1111/j.1365-313X.2011.04772.X

Kobayashi, T., Itai, R. N., Ogo, Y., Kakei, Y., Nakanishi, H., Takahashi, M., et al. (2009). The rice transcription factor IDEF1 is essential for the early response to iron deficiency, and induces vegetative expression of late embryogenesis abundant genes. Plant J. 60, 948-961. doi: 10.1111/j.1365-313X.2009. 04015.x

Kobayashi, T., Nagasaka, S., Senoura, T., Itai, R. N., Nakanishi, H., and Nishizawa, N. K. (2013). Iron-binding haemerythrin RING ubiquitin ligases regulate plant iron responses and accumulation. Nat. Commun. 4, 2792. doi: 10.1038/ncomms3792

Kobayashi, T., and Nishizawa, N. K. (2012). Iron uptake, translocation, and regulation in higher plants. Annu. Rev. Plant Biol. 63, 131-152. doi: 10.1146/annurevarplant-042811-105522

Kobayashi, T., and Nishizawa, N. K. (2014). Iron sensors and signals in response to iron deficiency. Plant Sci. 224, 36-43. doi: 10.1016/j.plantsci.2014.04.002

Kobayashi, T., Ogo, Y., Itai, R. N., Nakanishi, H., Takahashi, M., Mori, S., et al. (2007). The transcription factor IDEF1 regulates the response to and tolerance of iron deficiency in plants. Proc. Natl. Acad. Sci. U.S.A. 104, 19150-19155. doi: 10.1073/pnas.0707010104

Lan, P., Li, W., Wen, T.-N., Shiau, J.-Y., Wu, Y.-C., Lin, W., et al. (2011). iTRAQ protein profile analysis of Arabidopsis roots reveals new aspects critical for iron homeostasis. Plant Physiol. 155, 821-834. doi: 10.1104/pp.110.169508

Lanquar, V., Grossmann, G., Vinkenborg, J. L., Merkx, M., Thomine, S., and Frommer, W. (2014). Dynamic imaging of cytosolic zinc in Arabidopsis roots combining FRET sensors and RootChip technology. New Phytol. 202, 198-208. doi: 10.1111/nph.12652

Lee, J. W., and Helmann, J. D. (2007). Functional specialization within the Fur family of metalloregulators. Biometals 20, 485-499. doi: 10.1007/s10534-0069070-7

Long, T. A., Tsukagoshi, H., Busch, W., Lahner, B., Salt, D. E., and Benfey, P. N. (2010). The bHLH transcription factor POPEYE regulates response to iron deficiency in Arabidopsis roots. Plant Cell 22, 2219-2236. doi: 10.1105/tpc.110.074096

Poor, C. B., Wegner, S. V., Li, H., Dlouhy, A. C., Schuermann, J. P., Sanishvili, R., et al. (2014). Molecular mechanism and structure of the Saccharomyces cerevisiae iron regulator Aft2. Proc. Natl. Acad. Sci. U.S.A. 111, 4043-4048. doi: 10.1073/pnas.1318869111

Rampey, R. A., Woodward, A. W., Hobbs, B. N., Tierney, M. P., Lahner, B., Salt, D. E., et al. (2006). An Arabidopsis basic helix-loop-helix leucine zipper protein modulates metal homeostasis and auxin conjugate responsiveness. Genetics 174, 1841-1857. doi: 10.1534/genetics.106.061044

Römheld, V., and Marschner, H. (1986). Evidence for a specific uptake system for iron phytosiderophore in roots of grasses. Plant Physiol. 80, 175-180. doi: 10.1104/pp.80.1.175

Salahudeen, A. A., Thompson, J. W., Ruiz, J. C., Ma, H.-W., Kinch, L. N., Li, Q., et al. (2009). An E3 ligase possessing an iron-responsive hemerythrin domain is a regulator of iron homeostasis. Science 326, 722-726. doi: 10.1126/science. 1176326

Selote, D., Samira, R., Matthiadis, A., Gillikin, J. W., and Long, T. A. (2015). Iron-binding E3 ligase mediates iron response in plants by targeting bHLH transcription factors. Plant Physiol. 167, 273-286. doi: 10.1104/pp.114.250837

Stenkamp, R. E. (1994). Dioxygen and hemerythrin. Chem. Rev. 94, 715-726. doi: $10.1021 / \mathrm{cr} 00027 \mathrm{a} 008$

Thomine, S., and Vert, G. (2013). Iron transport in plants: better be safe than sorry. Curr. Opin. Plant Biol. 6, 1-6. doi: 10.1016/j.pbi.2013.01.003

Thompson, J. W., and Bruick, R. K. (2012). Protein degradation and iron homeostasis. Biochim. Biophys. Acta 1823, 1484-1490. doi: 10.1016/j.bbamcr.2012.02.003

Thompson, J. W., Salahudeen, A. A., Chollangi, S., Ruiz, J. C., Brautigam, C. A., Makris, T. M., et al. (2012). Structural and molecular characterization of ironsensing hemerythrin-like domain within F-box and leucine-rich repeat protein 5 (FBXL5). J. Biol. Chem. 287, 7357-7365. doi: 10.1074/jbc.M111.308684

Ueta, R., Fujiwara, N., Iwai, K., and Yamaguchi-Iwai, Y. (2012). Iron-induced dissociation of the Aftlp transcriptional regulator from target gene promoters is an initial event in iron-dependent gene suppression. Mol. Cell. Biol. 32, 4998-5008. doi: 10.1128/MCB.00726-12

Urzica, E. I., Casero, D., Yamasaki, H., Hsieh, S. I., Adler, L. N., Karpowicz, S. J., et al. (2012). Systems and trans-system level analysis identifies conserved iron deficiency responses in the plant lineage. Plant Cell 24, 3921-3948. doi: 10.1105/tpc.112.102491

Vashisht, A. A., Zumbrennen, K. B., Huang, X., Powers, D. N., Durazo, A., Sun, D., et al. (2009). Control of iron homeostasis by an iron-regulated ubiquitin ligase. Science 326, 718-721. doi: 10.1126/science.1176333

Conflict of Interest Statement: The authors declare that the research was conducted in the absence of any commercial or financial relationships that could be construed as a potential conflict of interest.

Copyright (c) 2015 Kobayashi and Nishizawa. This is an open-access article distributed under the terms of the Creative Commons Attribution License (CC BY). The use, distribution or reproduction in other forums is permitted, provided the original author(s) or licensor are credited and that the original publication in this journal is cited, in accordance with accepted academic practice. No use, distribution or reproduction is permitted which does not comply with these terms. 